\title{
Designing coupled-resonator optical waveguide delay lines
}

\author{
Joyce K. S. Poon, Jacob Scheuer, Yong Xu, and Amnon Yariv \\ Department of Electrical Engineering and Department of Applied Physics, California Institute of Technology, \\ MS 136-93, Pasadena, California 91125
}

Received December 18, 2003; revised manuscript received April 15, 2004; accepted April 30, 2004

\begin{abstract}
We address the trade-offs among delay, loss, and bandwidth in the design of coupled-resonator optical waveguide (CROW) delay lines. We begin by showing the convergence of the transfer matrix, tight-binding, and time domain formalisms in the theoretical analysis of CROWs. From the analytical formalisms we obtain simple, analytical expressions for the achievable delay, loss, bandwidth, and a figure of merit to be used to compare delay line performance. We compare CROW delay lines composed of ring resonators, toroid resonators, Fabry-Perot resonators, and photonic crystal defect cavities based on recent experimental results reported in the literature. (C) 2004 Optical Society of America
\end{abstract}

OCIS codes: $230.5750,230.7370,230.3120$.

\section{INTRODUCTION}

Optical microresonators have been attracting considerable theoretical and experimental attention because they have applications in fields ranging from fundamental physics to telecommunications systems. ${ }^{1}$ Resonators with effective volume $V \leqslant\left(\lambda_{0} / n\right)^{3}$ that possess only one electromagnetic mode in a given spectral region, such as the emission region of an inverted atomic population, have been essential for studies of atom-light interactions such as cavity quantum electrodynamics and the Purcell effect. ${ }^{1}$ Microresonator lasers may have low thresholds because both spontaneous emission and stimulated emission can take place in the same single electromagnetic mode. ${ }^{1}$ Resonators can also be used as optical filters and as add-drop multiplexers for optical communication systems. $^{2-4}$

A coupled-resonator optical waveguide (CROW) consists of a chain of resonators in which light propagates by virtue of the coupling between adjacent resonators. ${ }^{5-7}$ CROWs promise to be a new method for controlling the group velocity of optical pulses and thus potentially to find applications in storing and buffering optical pulses. ${ }^{8,9}$ Dispersion-related distortion in coupled-resonator structures was explored previously. ${ }^{7,10}$ In this paper we study the relations among the different theoretical treatments of CROWs, and we concentrate on the practical issues of this application of resonators, dealing with time delay, bandwidth, and loss.

The paper is organized as follows: In Section 2 we show the convergence of three theoretical frameworks, the tight-binding method, the transfer matrix method, and the temporal coupled-mode approach, using the specific example of ring resonators; in Section 3 the ring resonator example is generalized to Fabry-Perot and photonic crystal cavities; in Section 4 we derive several simple and intuitive equations for the delay, loss, and bandwidth of a CROW; in Section 5 we define a figure of merit of a CROW delay line that is generally applicable to resonator-based devices; in Section 6 we compare the performance of CROW delay lines made from various types of resonator, and we discuss their merits and demerits in Section 7.

\section{THEORETICAL APPROACHES}

The theory of CROWs and other coupled-resonator systems has been explored extensively in recent years. For a comprehensive understanding of CROWs, the assumptions that are inherent in and the relationships among the different theoretical approaches should be identified, so the appropriate model is used to study the problem at hand. The three formalisms most commonly used to study CROWs are the tight-binding approximation, ${ }^{5,11}$ the transfer matrix method, ${ }^{12-15}$ and temporal coupledmode theory. ${ }^{4,16}$ In this section we review these approaches and show that, whereas they may seem disparate at the outset, the three formalisms are consistent with one another in the limit of weak interresonator coupling.

To show the convergence of the theoretical approaches, we choose first to study a specific model of a sequence of ring resonators that are coupled serially in a phasematched manner as in Fig. 1 for the following reasons:

1. Ring resonators can be made to support a single transverse mode in a given spectral region, which is an essential feature in its practical and scientific applications. This property is in contrast to those of disk or spherical resonators with radii much greater than the optical wavelength.

2. Evanescent wave coupling between ring resonators and optical waveguides can be achieved straightforwardly and in planar geometry by simple lithographic techniques.

3. The simple modal structure and coupling mechanism facilitate an essentially exact analytical treatment of arbitrary sequences of coupled ring resonators and thus a meaningful comparison with experiments. 


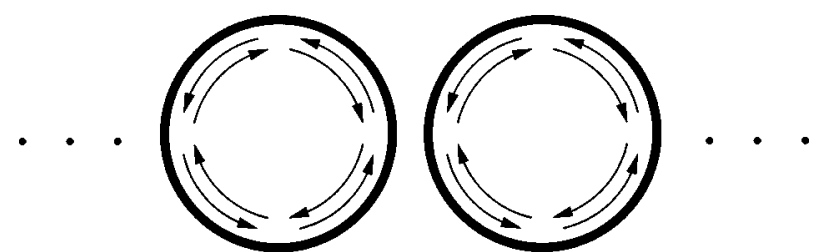

Fig. 1. Infinite chain of coupled ring resonators.

The results that we obtain from the analysis of the ring resonators will be generalized in Section 3 to photonic crystal and Fabry-Perot cavities.

\section{A. Tight-Binding Analysis}

An infinite CROW consisting of an arbitrary type of resonator can be analyzed by use of a tight-binding approach, in analogy to solid-state physics ${ }^{5,6,11}$ in which a propagating mode is expressed as an equiamplitude phase-locked excitation of the local mode of each resonator $\mathbf{E}_{\Omega}(\mathbf{r})$. In the limit of weak, nearest-neighbor interresonator coupling, dispersion relation $\omega(K)$ is

$$
\omega(K)=\Omega\left[1-\frac{\Delta \alpha}{2}+\gamma \cos (K \Lambda)\right],
$$

where $\Omega$ is the resonance frequency of the individual resonator, $K$ is the Bloch wave vector, $\Lambda$ is the periodicity, and $\Delta \alpha$ and $\gamma$ are

$$
\begin{aligned}
\Delta \alpha= & \int \mathrm{d}^{3} \mathbf{r}\left[\epsilon(\mathbf{r})-\epsilon_{0}(\mathbf{r})\right] \mathbf{E}_{\Omega}(\mathbf{r}) \cdot \mathbf{E}_{\Omega}(\mathbf{r}), \\
\gamma= & \int \mathrm{d}^{3} \mathbf{r}\left[\epsilon_{0}(\mathbf{r}-\Lambda \hat{\mathbf{z}})-\epsilon(\mathbf{r}-\Lambda \hat{\mathbf{z}})\right] \\
& \times \mathbf{E}_{\Omega}(\mathbf{r}) \cdot \mathbf{E}_{\Omega}(\mathbf{r}-\Lambda \hat{\mathbf{z}}) .
\end{aligned}
$$

$\epsilon(\mathbf{r})$ is the dielectric coefficient of the CROW and $\epsilon_{0}(\mathbf{r})$ is the dielectric coefficient of an individual resonator. ${ }^{5}$

The tight-binding model is mathematically elegant and applies to any kind of resonator. It has been used extensively in studies of both linear and nonlinear optical propagation in CROWs. ${ }^{10,17-19}$ This theoretical framework most readily lends itself to the analysis of infinitely long CROWs, or to those in which periodic boundary conditions apply, that consist of identical resonators.

\section{B. Transfer Matrix Analysis}

In practice, the number of coupled resonators in a CROW is finite and possibly not large; hence we need a designoriented analysis tool that can deal with any number of resonators $1 \leqslant N<\infty$. The transfer matrix approach $^{12-15}$ is particularly powerful in that it can deal with any arbitrary sequence of resonators and couplers, which is a prerequisite to general optical filter design. $^{2,4,20}$

For ring resonators, a special and important wave that can be excited is one in which each resonator supports a clockwise or a counterclockwise traveling wave with a sense of circulation opposite that of its immediate neighbors, as in Fig. 2. This circulating wave is not an eigenmode of the CROW but rather a superposition of the Bloch eigenmodes that consist of standing waves in each resonator shown in Fig. $1 .^{15}$ One can calculate the trans- mission through the CROW by successively multiplying a transfer matrix that characterizes the coupling between two adjacent rings and another matrix that accounts for the field propagation in the rings. Using the notation from Fig. 2, we describe the coupling region by the scattering matrix

$$
\left(\begin{array}{l}
a_{n+1} \\
b_{n+1}
\end{array}\right)=P\left(\begin{array}{l}
a_{n}{ }^{\prime} \\
b_{n}{ }^{\prime}
\end{array}\right), \quad P \equiv \frac{1}{\kappa}\left[\begin{array}{cc}
-t & 1 \\
-1 & t^{*}
\end{array}\right],
$$

where $t$ is the transmission coefficient, $\kappa$ is the dimensionless coupling coefficient over the coupling length, and $|t|^{2}+|\kappa|^{2}=1$ for lossless coupling. As the field propagates about the ring, it accumulates a phase shift and may be attenuated, such that

$$
\left(\begin{array}{l}
a_{n}{ }^{\prime} \\
b_{n}{ }^{\prime}
\end{array}\right)=Q\left(\begin{array}{l}
a_{n} \\
b_{n}
\end{array}\right), \quad Q \equiv\left[\begin{array}{cc}
0 & \exp (-i \pi \beta R) \\
\exp (i \pi \beta R) & 0
\end{array}\right] .
$$

$\beta$ is the propagation constant in the ring as given by $\beta$ $=n \omega / c$, where $n$ is the frequency-dependent effective index. $\quad \beta$ may include an imaginary part to account for loss or gain. Combining Eqs. (3) and (4), we have

$$
\left(\begin{array}{l}
a_{n+1} \\
b_{n+1}
\end{array}\right)=P Q\left(\begin{array}{l}
a_{n} \\
b_{n}
\end{array}\right) .
$$

Matrices $P$ and $Q$ can be specified at each frequency to account for any frequency dependence of the effective index, loss, and transmission and coupling coefficients. $P$ and $Q$ can also be specified differently for each resonator in the CROW to account for differences in resonator properties and coupling coefficients.

By successively multiplying $P$ and $Q$, we obtain a relation among the drop, through, and input ports shown in Fig. 2:

$$
\left(\begin{array}{l}
a_{N+1} \\
b_{N+1}
\end{array}\right) \equiv\left[\begin{array}{ll}
A & B \\
C & D
\end{array}\right]\left(\begin{array}{l}
a_{0} \\
b_{0}
\end{array}\right) .
$$

For a single input to the waveguide, $a_{0}, a_{N+1}=0$. Therefore the transfer functions at the through and the drop ports are, respectively,

$$
\begin{gathered}
\frac{b_{0}}{a_{0}}=-\frac{A}{B}, \\
\frac{b_{N+1}}{a_{0}}=C-\frac{A D}{B} .
\end{gathered}
$$

A key result of the transfer matrix approach is that it is consistent with the tight-binding method. Whereas the transfer matrices are outwardly quite different from the tight-binding analysis, the two methods give dispersion

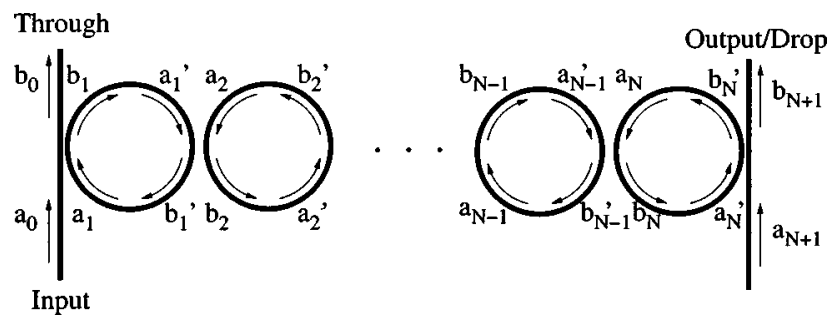

Fig. 2. Traveling wave in a finite chain of ring resonators. 
relations of the same form in the limit of weak coupling. As was detailed in Ref. 15, the dispersion relation as derived by application of the Bloch condition to the transfer matrices is

$$
\sin (\beta \pi R)= \pm \operatorname{Im}(\kappa) \cos (K \Lambda),
$$

where $K$ is the Bloch wave vector and $\Lambda$ is the periodicity of the structure. In the limit of weak coupling, $|\kappa| \ll 1$, the dispersion relation reduces to

$$
\omega(K)=\Omega\left[1 \pm \frac{|\kappa|}{m \pi} \cos (K \Lambda)\right],
$$

where $m=\Omega n R / c$ is the azimuthal modal number. Equation (9), derived from the transfer matrices, is of a form identical to that of Eq. (1) as derived from the tightbinding approximation with $\gamma$ in Eq. (1) replaced by $|\kappa| /(m \pi)$.

\section{Time Domain Analysis}

Another powerful approach in the study of coupledresonator systems is the time domain or temporal coupled-mode analysis. A time domain picture can also more easily facilitate the study of pulse propagation in the presence of certain optical nonlinearities, such as the Kerr effect. ${ }^{21,22}$ Little et al. previously analyzed coupled ring resonators by using temporal coupled-mode theory. ${ }^{4}$ We shall see that the transfer matrices, and hence the tight-binding model for an infinitely long chain, are also consistent with this approach.

As matrix analysis is a frequency-domain approach, the temporal dynamics is related to the transfer matrices by the Fourier transform. The field amplitudes in the matrix approach, $a_{n}$ and $b_{n}$, are the frequency-dependent Fourier components of the field. From the coupling matrices and for unidirectional, phase-matched coupling such that $\kappa^{*}=-\kappa,{ }^{23}$ by using Eqs. (3) and (4) we find that

$$
2 i a_{n} \sin (\beta \pi R)=i|\kappa|\left(a_{n+1}+a_{n-1}\right) .
$$

As $\beta=\omega n / c$ in the same way that Eq. (8) can be approximated by Eq. (9) in the limit of weak coupling, the lefthand side of Eq. (10) can be linearized such that

$$
i \omega a_{n}-i \Omega a_{n}=\frac{i|\kappa|(-1)^{m} \Omega}{2 m \pi}\left(a_{n+1}+a_{n-1}\right) .
$$

Taking the inverse Fourier transform of Eq. (11), we find that the evolution of the field in the time domain is

$$
\frac{\mathrm{d} \tilde{a}_{n}(t)}{\mathrm{d} t}-i \Omega \tilde{a}_{n}(t)=\frac{i(-1)^{m}|\kappa| \Omega}{2 m \pi}\left[\tilde{a}_{n+1}(t)+\tilde{a}_{n-1}(t)\right] .
$$

Attenuation or gain can be introduced by the addition of an imaginary part to $\Omega$. Substituting the form of the pulse envelope, $A_{n}(t)=\tilde{a}_{n}(t) \exp (-i \Omega t)$, into Eq. (12) yields

$$
\frac{\mathrm{d} A_{n}(t)}{\mathrm{d} t}=\frac{i(-1)^{m}|\kappa| \Omega}{2 m \pi}\left[A_{n+1}(t)+A_{n-1}(t)\right] .
$$

Equation (13) represents a set of linear first-order differential equations that can be solved for specific initial conditions. In analogy to an array of coupled waveguides, the solution to Eq. (13) for the initial conditions $A_{0}(t=0)=1$ and $A_{n \neq 0}(t=0)=0$ is

$$
A_{n}(t)=i^{n} J_{n}\left(\frac{t}{T_{\text {ext }}}\right),
$$

where $J_{n}$ is the $n$ th-order Bessel function and $T_{\text {ext }}$ $=(-1)^{m} m \pi /|\kappa| \Omega .^{23} \quad$ Appropriate superpositions of Bessel functions can be used to satisfy any arbitrary initial conditions and can thus describe the evolution of an arbitrary pulse in an infinite ring resonator CROW in a purely temporal picture.

Equation (12) is exactly identical to the result obtained previously by Little et al., whose analysis is based completely in the time domain. ${ }^{4}$ Reynolds et al. have also derived the same result for coupled defects in photonic crystals with nearest-neighbor coupling. ${ }^{16}$ That result shows that the transfer matrix method is identical to the temporal coupled-mode theory in the limit of weak, phasematched coupling. This equivalence is essential because it allows for the generalization of the results obtained by use of a particular approach to other structures for which that approach does not strictly apply.

The convergence of the time domain analysis, the tightbinding approximation, and the transfer matrices is of fundamental interest, as it illustrates that these different points of view are consistent with one another within the inherent assumptions of the models and in the limit of weak coupling.

\section{PHOTONIC CRYSTAL DEFECT AND FABRY-PEROT CAVITIES}

Even though our derivations have been based on the example of ring resonators thus far, in this section we consider ways in which to generalize the ring resonator results to two other important classes of resonator: the photonic crystal defect and Fabry-Perot cavities.

Because the modes of photonic crystal defect cavities cannot be readily decomposed into traveling plane waves, the analysis of coupled photonic crystal defect cavities by transfer matrix methods has been limited. ${ }^{24,25}$ However, because the temporal coupled-mode equations for the ring resonator and the photonic crystal defect cavity CROWs are identical, ${ }^{16}$ the conclusions that we draw from the ring resonator example hold for the latter case with $|\kappa| /(m \pi)$ replaced $\gamma$ in Eq. (2b).

The Fabry-Perot CROWs as in Fig. 3 can be analyzed in the same way as the photonic crystal defect cavities as well as by use of transfer matrices. The coupling will be determined by the reflection and transmission coefficients of the end mirrors of each cavity. ${ }^{26}$ However, coupling coefficient $\kappa$ is generally complex, not imaginary, in the case of phase-matched codirectional coupling as derived from coupled-mode theory. ${ }^{23,26}$ Moreover, because FabryPerot resonators are one dimensional, they can be completely described by $2 \times 2$ transfer matrices, ${ }^{26}$ in contrast to ring resonators, which require $4 \times 4$ transfer matrices for modeling the Bloch modes. ${ }^{15}$ Therefore there are only two Bloch modes at each frequency rather than four as for the ring resonator CROW. 


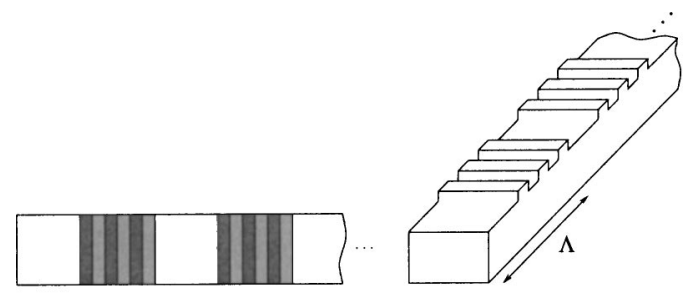

(a)

(b)

Fig. 3. Coupled Fabry-Perot cavities. (a) Epitaxilly grown; (b) gratings on a waveguide.

For a general complex coupling coefficient between the Fabry-Perot (one-dimensional) cavities, $\kappa=\kappa_{0} \exp (i \theta)$, the dispersion relation becomes

$$
\frac{\omega(K)}{\Omega}=1+\frac{(-1)^{m} \kappa_{0}}{m \pi}[\sin (K \Lambda-\theta)] .
$$

The dispersion relation is of the same form as Eq. (9), but the \pm is absent and $\theta$ can be arbitrarily specified, depending on the nature of the coupling. Whereas it does not significantly alter the general characteristics of a CROW, the presence of the phase shift modifies the phase velocity of the CROW Bloch modes. Nonetheless, the results discussed thus far for the ring CROW still hold for a chain of coupled linear resonators with $\pi R$ replaced by $L$, the length of the cavity.

\section{DELAY, LOSS, AND BANDWIDTH}

Using dispersion relation (9) and the transfer matrices for the specific case of ring resonators, we now proceed to derive and verify the expressions that highlight the tradeoffs among delay, bandwidth, and loss of a CROW. As discussed in Section 3, the results that we obtain can easily be generalized to other common types of resonator. The immediate consequence of Eq. (9) is that group velocity $\left|v_{g}\right| \equiv \mathrm{d} \omega / \mathrm{d} K=\Lambda \Omega|\kappa /(m \pi) \sin (K \Lambda)|$ is dependent on coupling coefficient $|\kappa|$. As $|\kappa|$ can be controlled by the separation between adjacent resonators, we can in principle achieve arbitrarily large slowing down of optical pulses. We can also control the delay by changing the refractive index of the resonators or the coupling region through the electro-optic or thermo-optic effect. ${ }^{27-30}$ However, as $|\kappa|$ decreases, so does the bandwidth of the CROW, and the overall loss of the CROW becomes more sensitive to the intrinsic losses in the individual resonator. The latter effect occurs because the light spends more time in a resonator before tunneling to its neighbor.

In the absence of other mechanisms such as Kerr nonlinearity ${ }^{21,22}$ to compensate for group-velocity dispersion, an optical pulse propagating in a CROW should have a central frequency near the zero GVD region of the dispersion curve $\left(\partial^{2} K / \partial \omega^{2} \approx 0\right)$ to minimize the accumulated distortion. This condition occurs at the center frequency, $\omega \approx \Omega$, where the group velocity is maximum, $v_{g, \max }=|\kappa| \Omega \Lambda /(m \pi)$. From Eq. (9), a CROW band spans the frequency range $\Delta \omega=2 \Omega|\kappa| /(m \pi)$. Consequently we define the usable bandwidth of a CROW as half of this total bandwidth centered at $\Omega$ :

$$
\Delta \omega_{\mathrm{use}} \equiv \frac{|\kappa| c}{\pi n R} .
$$

The periodicity, $\Lambda$, is taken to be approximately equal to $2 R$. Thus the temporal delay of a pulse propagating through the whole length of the CROW is determined by the distance traversed in the CROW and the group velocity at $\Omega$ :

$$
\tau=\frac{\pi n R N}{|\kappa| c} .
$$

From Eq. (17) we observe that the CROW effectively acts as a conventional waveguide with group velocity $c / n$ but with a length of

$$
L_{\mathrm{eff}}=\frac{c \tau}{n}=\frac{\pi R N}{|\kappa|},
$$

i.e., $\sim 1 /|\kappa|$ times longer than the CROW. Because of the reduced group velocity provided by the feedback among the coupled resonators, the total length of the CROW, $N \Lambda$, is contracted by a factor of $S=(\pi / 2)|\kappa| . \quad S$ represents the slowing factor of the group velocity, $c /\left(n v_{g, \max }\right)$. The contraction in the spatial length also applies to a pulse propagating through the structure, such that a 100-ps pulse in a fiber, which has a spatial extent of $\sim 2$ $\mathrm{cm}$, contracts to a length of $\sim 3 \mathrm{~mm}$ in a CROW with $|\kappa|$ $=0.1$.

Furthermore, the loss from the input to the output of the CROW is intuitively given by the product of the loss per unit length and $L_{\text {eff }}$ :

$$
\alpha=\frac{a \pi R N}{|\kappa|}=\frac{m \pi N}{|\kappa| Q_{\mathrm{int}}},
$$

where $\exp (-\alpha)$ is the net power attenuation coefficient of the CROW, $\exp (-a 2 \pi R)$ is the power attenuation in the waveguides of the constituent resonators, and $Q_{\text {int }}$ is the intrinsic quality factor or $Q$ factor of the resonator. Equations (16), (17), and (19) facilitate the straightforward design of CROW delay lines.

If only a specific loss is tolerated, the maximal delay achievable is independent of $|\kappa|$. To illustrate this, we define the maximum tolerable loss as $\exp (-\alpha)=\exp (-1)$. Using Eqs. (19) and (17), we find that

$$
\tau_{\max }=n / c a \text {. }
$$

This result makes intuitive sense because light must travel the same optical length to achieve a given delay. The role of the resonators is now clear: The weakly coupled resonators make this net length more compact.

To verify the simple and intuitive equations, Eqs. (16), (17), and (19), we compare them with numerical results obtained from the transfer matrices. In coupledresonator filter synthesis, the coupling constants must be at specific ratios to prevent ripples in the passband, which cause significant deviations from the ideal, infinite CROW characteristics. ${ }^{4,31,32}$ For a maximally flat transfer function, the interresonator couplings are not constant throughout the structure, ${ }^{4,32,33}$ hence Eqs. (16), (17), and (19) do not strictly apply. However, we may still obtain fairly flat transfer functions over $\Delta \omega_{\text {use }}$ by having a single interresonator coupling constant, $\kappa$, and a different 
waveguide-resonator coupling, $\kappa_{i}$. Figure 4 shows the passband spectra for a finite CROW with a single $\kappa$ and a different $\kappa_{i}$. As shown in Fig. 4 one pair of $\kappa$ and $\kappa_{i}$ is sufficient to produce a flat transmission spectrum over a large range in the number of resonators. The flat responses enable us to use finite structures to mimic an infinitely long CROW characterized by a single coupling constant.

Therefore, using the parameters in Table 1 when $N$ is fixed to be 10 and $\kappa$ is varied, and setting $\kappa_{i}=-0.43 i$ and $\kappa=-0.1 i$ when $N$ is varied, we compare Eqs. (16), (17), and (19) with the calculations from the transfer matrices. As shown in Fig. 5, the expressions are in excellent agreement with the numerical calculations. In the plots of the delay times, the theoretical results as indicated by the solid curves in Fig. 5 coincide most closely with the data points that correspond to slight waveguide losses $(\sim 4 \mathrm{~dB} /$ $\mathrm{cm})$. This occurs because the losses smooth out the transmission spectrum ripples, making the passband of the finite CROW a better approximation to an infinitely long CROW.

\section{FIGURE OF MERIT}

To compare CROW delay lines composed in a general way, independently of material systems and resonator sizes, we need a benchmark with which to determine the quality of a delay line. Although $\tau_{\max }$ is a useful criterion, it

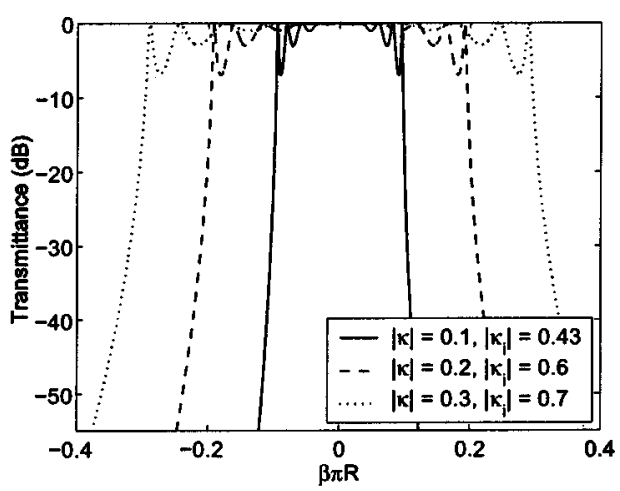

(a) $\mathrm{N}=10$

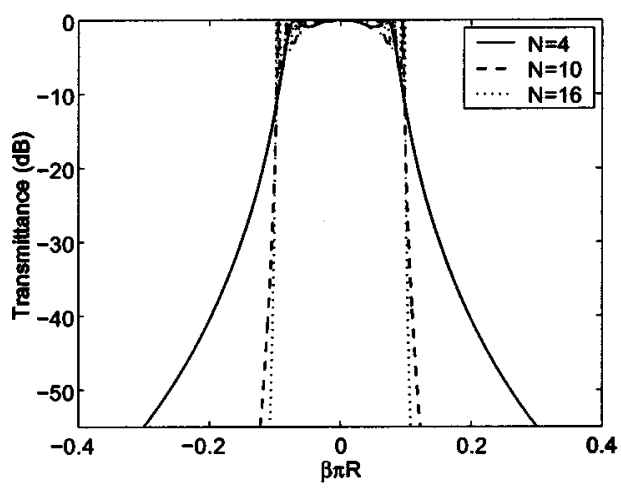

(b) $|\kappa|=0.1,\left|\kappa_{i}\right|=0.43$

Fig. 4. Passbands of coupled-resonator structures with identical interresonator coupling $|\kappa|$ throughout and different waveguideresonator coupling $\left|\kappa_{i}\right|$. (a) The number of resonators is fixed and the number of coupling constants is varied. (b) The number of coupling constants is fixed and $N$ is varied.
Table 1. $|\kappa|$ and the Corresponding $\left|\kappa_{i}\right|$ Used in Fig. 5 for $N=10$

\begin{tabular}{lllllll}
\hline \multicolumn{7}{c}{ Coupling Coefficient } \\
\hline$|\kappa|$ & 0.1 & 0.15 & 0.2 & 0.25 & 0.3 & 0.35 \\
$\left|\kappa_{i}\right|$ & 0.43 & 0.55 & 0.6 & 0.65 & 0.7 & 0.75 \\
\hline
\end{tabular}

does not account for the usable bandwidth. An alternative approach is to compare the intrinsic and coupling losses in the resonators. The intrinsic losses of each resonator that are due to absorption and scattering are characterized by a time $T_{\text {int }}$. The decay of resonator power caused by coupling to adjacent neighbors is characterized by $T_{\text {ext }}$ and the associated $Q, Q_{\text {ext }}=\Omega T_{\text {ext }}$.

$\left|T_{\text {ext }}\right|$, from Eq. (14), naturally defines the characteristic lifetime of the excitation that is due to the coupling, as it sets the temporal width of the field in the initially excited resonator. It is also the time required for a pulse centered at $\Omega$ to traverse a single resonator, i.e., $\left|T_{\text {ext }}\right|$ $=\Lambda / v_{g, \max }$. At $t=\left|T_{\text {ext }}\right|$ the energy at the zeroth resonator will have decayed to $\left|J_{0}(1)\right|^{2} \approx 0.59$ of its original value.

To be useful as a delay line, $T_{\text {ext }} \ll T_{\text {int }}$ or equivalently $Q_{\text {ext }} \ll Q_{\text {int }}$. Therefore a useful figure of merit (FOM) for CROW delay lines is

$$
\mathrm{FOM} \equiv Q_{\text {int }} / Q_{\text {ext }} .
$$

The figure of merit is also useful for other resonator-based devices or geometries for comparing the relative roles of $Q_{\text {int }}$ and $Q_{\text {ext }}$, for example, in the determination of the loaded $Q, 1 / Q_{L}=1 / Q_{\text {int }}+1 / Q_{\text {ext }}$.

Substituting $\left|T_{\text {ext }}\right|=m \pi /|\kappa| \Omega$ from Eq. (14) into $Q_{\text {ext }}$ $=\Omega\left|T_{\text {ext }}\right|$, we find that

$$
Q_{\text {ext }}=m \pi /|\kappa| \text {. }
$$

Figure of merit (21), in turn, simplifies to

$$
\mathrm{FOM}=\frac{|\kappa|}{a \pi R}=\frac{\Delta \omega_{\mathrm{use}} \tau}{\alpha_{\mathrm{tot}}}=\tau_{\max } \Delta \omega_{\mathrm{use}} .
$$

The second equality reveals that the figure of merit can alternatively be viewed as a balancing of bandwidth, loss, and delay.

\section{COMPARING DIFFERENT RESONATORS}

Table 2 compares 10-resonator-long delay lines with $|\kappa|$ $=0.1$ ( $1 \%$ power coupling) composed of resonators in different material systems at $1.55 \mu \mathrm{m}$. The results emphasize the trade-offs between delay and bandwidth. For the semiconductor, polymer, and Hydex ring resonators and the photonic crystal cavities, we use some of the highest experimental $Q_{\text {int }}$ values of a single, passive resonator reported in the literature to date and their corresponding resonator sizes. ${ }^{34-36,38,39}$

A particular issue with ultrahigh- $Q$ resonators is that excess coupling losses may become dominant over intrinsic resonator $Q$. The source of the excess coupling loss is that the neighboring resonators act as a dielectric perturbation to an individual resonator. In the ring microresonators with lower $Q_{\text {int }}$ values, bending and scattering losses typically predominate. ${ }^{40}$ Because the coupling is 


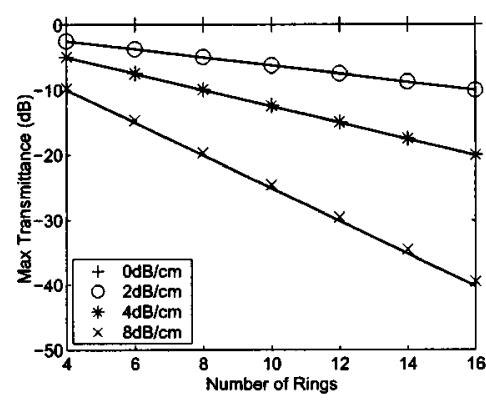

(a) Transmittance vs. $N$ for $|\kappa|=0.1$.

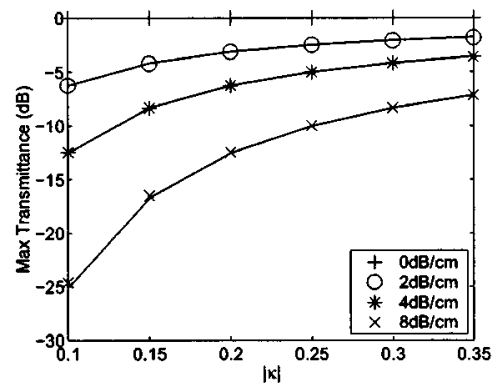

(c) Transmittance vs. $|\kappa|$ for $N=10$.

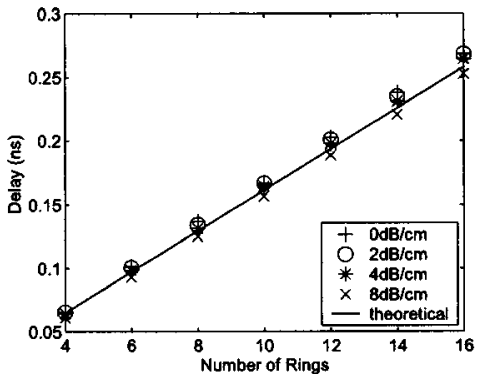

(b) Delay vs. $N$ for $|\kappa|=0.1$.

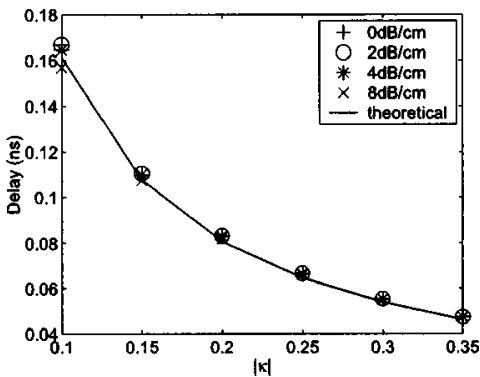

(d) Delay vs. $|\kappa|$ for $N=10$.

Fig. 5. Comparing analytical expressions for loss and delay with numerical results by use of the transfer matrices for various propagation losses in the resonators. Solid curves, theoretical results; markers, numerical results. $R=100 \mu \mathrm{m}$ and $n=1.54$.

Table 2. Comparison of CROW Delay Lines Consisting of $N=10$ Resonators for $|\kappa|=0.1$

\begin{tabular}{|c|c|c|c|c|c|c|}
\hline Type of Resonator & $Q_{\text {int }}$ & Net Loss $(\mathrm{dB})$ & Delay & Bandwidth & $\tau_{\max }$ & Figure of Merit \\
\hline $\begin{array}{l}\text { Semiconductor ring } \\
\qquad(n \sim 3, R \sim 10 \mu \mathrm{m})^{\mathrm{a}}\end{array}$ & 5000 & 33 & $31 \mathrm{ps}$ & $51 \mathrm{GHz}$ & $4 \mathrm{ps}$ & 1.3 \\
\hline $\begin{array}{l}\text { Polymer ring } \\
\qquad(n \sim 1.57, R \sim 32 \mu \mathrm{m})^{\mathrm{b}}\end{array}$ & $2.4 \times 10^{4}$ & 12 & $53 \mathrm{ps}$ & $30 \mathrm{GHz}$ & $20 \mathrm{ps}$ & 3.8 \\
\hline $\begin{array}{l}\text { Hydex ring } \\
\qquad(n \sim 1.6, R=40 \mu \mathrm{m})^{\mathrm{c}}\end{array}$ & $4 \times 10^{5}$ & 0.9 & $67 \mathrm{ps}$ & $24 \mathrm{GHz}$ & $329 \mathrm{ps}$ & 49 \\
\hline $\begin{array}{l}\text { Fiber ring } \\
\quad(n=1.45, R=10 \mathrm{~cm})^{\mathrm{d}}\end{array}$ & $8 \times 10^{7}$ & 10 & $152 \mathrm{~ns}$ & $11 \mathrm{MHz}$ & $66 \mathrm{~ns}$ & 4.3 \\
\hline $\begin{array}{l}\text { Silica toroid } \\
\quad(n \sim 1.45, R=60 \mu \mathrm{m})^{\mathrm{e}}\end{array}$ & $10^{8}$ & $5 \times 10^{-3}$ & $91 \mathrm{ps}$ & $17 \mathrm{GHz}$ & $82 \mathrm{~ns}$ & 9026 \\
\hline $\begin{array}{l}\text { Semiconductor Fabry-Perot } \\
\quad(n \sim 3, L=30 \mu \mathrm{m})\end{array}$ & $2 \times 10^{6}$ & 0.06 & $30 \mathrm{ps}$ & $53 \mathrm{GHz}$ & $2.2 \mathrm{~ns}$ & 724 \\
\hline $\begin{array}{l}\text { Photonic crystal defect } \\
\qquad\left(\gamma=2 \times 10^{-4}\right)^{\mathrm{f}}\end{array}$ & $4.5 \times 10^{4}$ & 5 & $41 \mathrm{ps}$ & $39 \mathrm{GHz}$ & $37 \mathrm{ps}$ & 9 \\
\hline
\end{tabular}

${ }^{a}$ Ref. 34 .

${ }^{b}$ Ref. 35

${ }^{c}$ Ref. 36.

${ }^{d}$ Includes excess coupling losses.

${ }^{e}$ Ref. 37, neglects excess coupling losses.

${ }^{f}$ Refs. 38 and 39.

assumed to be lossless in our analysis, the excess loss of the coupler should be accounted for by the loss of the resonator. Therefore, for a fiber ring resonator, in which material, bending, and splice losses are negligible, the loss in calculating the $Q$ factor in Table 2 is taken to be the excess loss of a commercial fused fiber coupler $[\sim 0.2 \mathrm{~dB}$ (Ref. 41)].

As no excess coupling loss values for ultrahigh- $Q$ toroid resonators have been reported to date, we neglect this effect in Table 2 and simply use the highest reported intrinsic $Q$ value $\left(Q_{\text {int }} \approx 10^{8}\right)$ of a single resonator. ${ }^{37,42}$ Ex- trapolating from this $Q$ value, the loss per revolution inside the toroid resonator is $\sim 10^{-4} \mathrm{~dB}$. For excess coupling loss to be negligible, it must be $\ll 10^{-4} \mathrm{~dB}$. Even for an excess coupling loss of $0.01 \mathrm{~dB}$, the $Q$ value in our comparison drops to $10^{6}$, the net loss increases to $0.5 \mathrm{~dB}, \tau_{\max }$ becomes $0.82 \mathrm{~ns}$, and the figure of merit is reduced to 90 . However, the coupling between a fiber taper and an ultrahigh- $Q$ silica microsphere has been experimentally shown to be nearly (>99.97\%) lossless ${ }^{43}$; therefore the coupling of toroid resonators may be nearly ideal as well.

CROWs based on Fabry-Perot cavities with an opera- 
tional wavelength near $600 \mathrm{~nm}$ were recently demonstrated. ${ }^{44}$ The coupling between Fabry-Perot resonators can be controlled by Bragg reflection. Hence, to compare this structure with the ring resonators for which the coupling is due to evanescent decay of the field outside the resonator, one takes the resonator $Q$ of the coupled Fabry-Perot structure not as the $Q$ of the composite structure of the cavity and Bragg layers but rather as the cavity by itself. The loss is thus determined by material and waveguide loss, which is taken to be 0.2 $\mathrm{dB} / \mathrm{cm}$ for the comparison, assuming that the Bragg gratings are etched on a waveguide. The losses at the coupling regions are ignored.

Experimental progress in photonic crystal coupledcavity structures has been burgeoning over the past few years. ${ }^{9,16,45-48}$ To compare photonic crystal defect cavity CROWs with the ring and Fabry-Perot resonators we take $\gamma$ to be $2 \times 10^{-4}$, which is approximately equal to the value of $|\kappa| /(m \pi)$ for the other integrated optical resonators considered.

\section{DISCUSSION}

As evidenced by the comparison in Table 2, application requirements, such as for the loss, bandwidth, and material system, dictate the type of resonator that will be the most suitable. To achieve long delays without too much attenuation requires low-loss (high- $Q$ ) resonators. High- $Q$ resonators also allow for more flexibility in the design because the CROW can be made longer with a higher coupling coefficient to increase the bandwidth without severely exacerbating the loss. For our example of $|\kappa|$ $=0.1$ and cavity lengths of tens of micrometers, for substantial delay with an attenuation of $\sim 10 \mathrm{~dB}, Q$ values of $10^{5}-10^{6}$ are necessary.

Although the use of ultrahigh- $Q$ resonators for a CROW is an attractive option, there have not been many reported experimental demonstrations of coupling of more than a few of these resonators together. ${ }^{49}$ Moreover, thus far there have not been extensive attempts to integrate these types of resonator with other planar components. Therefore, with the technological and experimental progress to date, a more practical approach may be to use resonators with lower- $Q$ values as the constituent elements of a CROW.

Ring resonators and Fabry-Perot resonators are promising for the realization of CROWS. Fabry-Perot cavities have the advantage that the coupling can be precisely controlled by Bragg reflection. However, Fabry-Perot resonators fabricated by epitaxial growth or thin-film deposition cannot be readily integrated with planar technologies. A lithographically defined grating on a waveguide requires a multistep fabrication process that has already been well exploited in distributed-feedback and distributed Bragg reflector structures.

Even though the patterning of gratings is well established, the option of using ring resonators should not be neglected. Ring resonators are more compact than a linear chain of Fabry-Perot resonators and can be fabricated in planar integrated light-wave circuits in a single lithographic step in the case of horizontal coupling. Recently, loss-compensated ring resonators in GaInAsP-InP were reported ${ }^{50}$; thus a lossless or even an amplifying microring CROW may be feasible. As CROWs are typically narrowband devices, even slight deviations in resonator sizes will alter the passband spectrum in the form of the Vernier effect. ${ }^{31,32}$ To achieve ideal device behavior, postfabrication tuning of the resonators through UV trimming, ${ }^{51,52}$ the thermo-optic effect, ${ }^{53}$ or the electrooptic effect ${ }^{35}$ maybe required. However, there have already been impressive demonstrations of flat-passband, low-loss, high-order (up to 11th-order) coupled microring resonator filters, ${ }^{36}$ illustrating that the coupled ring resonators may indeed be a practical building block for integrated optical devices. Recent advances in the fabrication of ring resonators continue to facilitate the achievement of passive and active ring resonator CROW delay lines in integrated optics.

\section{CONCLUSION}

In summary, we have addressed a number of key issues in designing CROW delay lines made from ring resonators. We have shown the convergence of the tight-binding approximation, the transfer matrix method, and the temporal mode coupling analysis. Achievable delay, available bandwidth, and loss are given by simple, analytical expressions that are in excellent agreement with numerical results from the transfer matrix method. We have proposed a figure of merit with which to compare various CROW delay lines that is a ratio between the lifetime of an individual resonator and the lifetime that is due to resonator coupling. This comparison offers an easy and quick gauge for determining the feasibility of and the minimum intrinsic resonator $Q$ necessary for a CROW delay line. We examined experimental progress in optical resonators to find that $\mathrm{CROW}$ delay lines with bandwidths of $\sim 50 \mathrm{GHz}$ and delays of the order of $100 \mathrm{ps}$ should be feasible with current technologies.

\section{ACKNOWLEDGMENTS}

J. Poon thanks K. J. Vahala, G. T. Paloczi, and W. W. Ng for fruitful discussions. She is grateful for the support of the Natural Sciences and Engineering Research Council of Canada. The financial support of Hughes Research Laboratories, the National Science Foundation, and the Defense Advanced Research Projects Agency is gratefully acknowledged.

J. K. S. Poon's e-mail address' is poon@caltech.edu.

\section{REFERENCES AND NOTES}

1. K. J. Vahala, "Optical microcavities," Nature (London) 424, 839-846 (2003).

2. C. K. Madsen, "General IIR optical filter design for WDM applications using all-pass filters," J. Lightwave Technol. 18, 860-868 (2000).

3. B. E. Little, S. T. Chu, W. Pan, D. Ripin, T. Kaneko, Y. Kokubun, and E. Ippen, "Vertically coupled glass microring resonator channel dropping filters," IEEE Photonics Technol. Lett. 11, 215-217 (1999).

4. B. E. Little, S. T. Chu, H. A. Haus, J. Foresi, and J.-P. Laine, "Microring resonator channel dropping filter," J. Lightwave Technol. 15, 998-1005 (1997).

5. A. Yariv, Y. Xu, R. K. Lee, and A. Scherer, "Coupled- 
resonator optical waveguide: a proposal and analysis," Opt. Lett. 24, 711-713 (1999).

6. N. Stefanou and A. Modinos, Impurity bands in photonic insulators, Phys. Rev. B 57, 12127-12133 (1998).

7. A. Melloni and F. Morichetti, "Linear and nonlinear pulse propagation in coupled resonator slow-wave optical structures," Opt. Quantum Electron. 35, 365-379 (2003).

8. G. Lenz, B. J. Eggleton, C. K. Madsen, and R. E. Slusher, "Optical delay lines based on optical filters," IEEE J. Quantum Electron. 37, 525-532 (2001).

9. S. Lan, S. Nishikawa, H. Ishikawa, and O. Wada, "Engineering photonic crystal impurity bands for waveguides, all-optical switches and optical delay lines," IEICE Trans. Electron. E85C, 181-189 (2002).

10. S. Mookherjea and A. Yariv, "Pulse propagation in a coupled-resonator optical waveguide to all orders of dispersion," Phys. Rev. E 65, 056601 (2002).

11. M. Bayindir, B. Temelkuran, and E. Ozbay, "Tight-binding description of the coupled defect modes in threedimensional photonic crystals," Phys. Rev. Lett. 84, 2140$2143(2000)$

12. K. Oda, N. Takato, and H. Toba, "A wide-FSR waveguide double-ring resonator for optical FDM transmission systems," J. Lightwave Technol. 9, 728-736 (1991).

13. R. Orta, P. Savi, R. Tascone, and D. Trinchero, "Synthesis of multiple-ring resonator filters for optical systems," IEEE Photonics Technol. Lett. 7, 1447-1449 (1995).

14. G. T. Paloczi, Y. Huang, A. Yariv, and S. Mookherjea, "Polymeric Mach-Zehnder interferometer using serially coupled microresonators," Opt. Express 11, 2666-2671 (2003), http://www.opticsexpress.org.

15. J. K. S. Poon, J. Scheuer, S. Mookherjea, G. T. Paloczi, Y. Huang, and A. Yariv, "Matrix analysis of microring coupledresonator optical waveguides," Opt. Express 12, 90-103 (2004), http://www.opticsexpress.org.

16. A. L. Reynolds, U. Peschel, F. Lederer, P. J. Roberts, T. F. Krauss, and P. J. I. de Maagt, "Coupled defects in photonic crystals," IEEE Trans. Microwave Theory Tech. 49, 1860 1867 (2001)

17. S. Mookherjea and A. Yariv, "Optical pulse propagation in the tight-binding approximation," Opt. Express 9, 91-96 (2001), http://www.opticsexpress

18. S. Mookherjea and A. Yariv, "Optical pulse propagation and holographic storage in a coupled-resonator optical waveguide," Phys. Rev. E 64, 066602 (2001).

19. S. Mookherjea and A. Yariv, "Second-harmonic generation with pulses in a coupled-resonator optical waveguide," Phys. Rev. E 65, 026607 (2002)

20. J. V. Hryniewicz, P. P. Absil, B. E. Little, R. A. Wilson, and P.-T. Ho, "Higher order filter response in coupled microring resonators," IEEE Photonics Technol. Lett. 12, 320-322 (2000).

21. D. N. Christodoulides and N. K. Efremidis, "Discrete temporal solitons along a chain of nonlinear coupled microcavities embedded in photonic crystals," Opt. Lett. 27, 568-570 (2002).

22. S. Mookherjea and A. Yariv, "Kerr-stabilized super-resonant modes in coupled-resonator optical waveguides," Phys. Rev. E 66, 046610 (2002)

23. A. Yariv, Optical Electronics in Modern Communications, 5th ed. (Paren Oxford U. Press, New York, 1997).

24. O. J. Painter, K. Srinivasan, and P. E. Barclay, "Wannierlike equation for the resonant cavity modes of locally perturbed photonic crystals," Phys. Rev. B 68, 035214 (2003).

25. Z.-Y. Li and K.-M. Ho, "Light propagation in semi-infinite photonic crystals and related waveguide structures," Phys. Rev. B 68, 155101 (2003)

26. A. Yariv and P. Yeh, Optical Waves in Crystals: Propagation and Control of Laser Radiation (Wiley, New York, 1984).

27. P. Rabiei and W. H. Steier, "Tunable polymer double microring filters," IEEE Photonics Technol. Lett. 15, 1255-1257 (2003).

28. Y. Yanagase, S. Yamagata, and Y. Kokubun, "Wavelength tunable polymer microring resonator filter with $9.4 \mathrm{~nm}$ tuning range," Electron. Lett. 39, 922-924 (2003).
29. R. L. Dantec, T. Benyattou, G. Guillot, A. Spisser, C. Seassal, J. L. Leclercq, P. Viktorovitch, D. Rondi, and R. Blondeau, "Tunable microcavity based on InP-air Bragg mirrors," IEEE J. Sel. Top. Quantum Electron. 5, 111-114 (1999).

30. M. Strassner, C. Luber, A. Tarraf, and N. Chitica, "Widely tunable-constant bandwidth monolithic Fabry-Perot filter with a stable cavity design for WDM systems," IEEE Photonics Technol. Lett. 14, 1548-1550 (2002).

31. C. K. Madsen and J. H. Zhao, Optical Filter Design and Analysis: A Signal Processing Approach (Wiley, New York, 1999).

32. A. Melloni and M. Martinelli, "Synthesis of direct-coupledresonators bandpass filters for WDM systems," J. Lightwave Technol. 20, 296-303 (2002).

33. H. F. Taylor, "Design of multireflector resonant bandpass filters for guided wave optics," J. Lightwave Technol. 19, 866-871 (2001).

34. R. Grover, V. Van, T. A. Ibrahim, P. P. Absil, L. C. Calhoun, F. G. Johnson, J. V. Hryniewicz, and P.-T. Ho, "Parallelcascaded semiconductor microring resonators for highorder and wide-FSR filters," J. Lightwave Technol. 20, 900905 (2002).

35. P. Rabiei, W. H. Steier, C. Zhang, and L. R. Dalton, "Polymer micro-ring filters and modulators," J. Lightwave Tech nol. 20, 1968-1975 (2002).

36. P. P. Absil, S. T. Chu, D. Gill, J. V. Hryniewicz, F. Johnson, O. King, B. E. Little, F. Seiferth, and V. Van, "Very high order integrated optical filters," in Optical Fiber Communication Conference (OFC), Vol. 95 of OSA Trends in Optics and Photonics Series (Optical Society of America, Washington, D.C., 2004), paper TuL3.

37. D. K. Armani, T. J. Kippenberg, S. M. Spillane, and K. J. Vahala, "Ultra-high-Q toroid microcavity on a chip," Nature (London) 421, 925-928 (2003).

38. Y. Akahane, T. Asano, B. S. Song, and S. Noda, "High-Q photonic nanocavity in a two-dimensional photonic crystal," Nature (London) 425, 944-947 (2003).

39. K. Srinivasan, P. E. Barclay, O. J. Painter, J. Chen, A. Y. Cho, and C. Gmachl, "Experimental demonstration of a high quality factor photonic crystal microcavity," Appl. Phys. Lett. 83, 1915-1917 (2003)

40. S. C. Hagness, D. Rafizadeh, S. T. Ho, and A. Taflove "FDTD microcavity simulations: design and experimental realization of waveguide-coupled single-mode ring and whispering-gallery-mode disk resonators," J. Lightwave Technol. 15, 2154-2165 (1997).

41. For example specifications of the JDS fused coupler, polarization maintaining tap are provided on the JDS Uniphase website: www.jdsu.com/site/images/products/pdf/ FFP_021604.pdf

42. A. L. Martin, D. K. Armani, L. Yang, and K. J. Vahala "Replica-molded high- $Q$ polymer microresonators," Opt. Lett. 29, 533-535 (2004).

43. S. M. Spillane, T. J. Kippenberg, O. J. Painter, and K. J. Vahala, "Ideality in a fiber-taper-coupled microresonator system for application to cavity quantum electrodynamics," Phys. Rev. Lett. 91, 043902 (2003).

44. M. Bayindir, S. Tanriseven, and E. Ozbay, "Propagation of light through localized coupled-cavity modes in onedimensional photonic band-gap structures," Appl. Phys. A: Solids Surf. 72, 117-119 (2001)

45. S. Olivier, C. Smith, M. Rattier, H. Benisty, C. Weisbuch, T. Krauss, R. Houdre, and U. Osterle, "Miniband transmission in a photonic crystal waveguide coupled-resonator optical waveguide," Opt. Lett. 26, 1019-1051 (2001).

46. T. J. Karle, D. H. Brown, R. Wilson, M. Steer, and T. F. Krauss, "Planar photonic crystal coupled cavity waveguides," IEEE J. Sel. Top. Quantum Electron. 8, 909918 (2002).

47. S. Nishikawa, S. Lan, N. Ikeda, Y. Sugimoto, H. Ishikawa, and K. Asakawa, "Optical characterization of photonic crystal delay lines based on one-dimensional coupled defects," Opt. Lett. 27, 2079-2081 (2002).

48. T. D. Happ, M. Kamp, A. Forchel, J. L. Gentner, and L. 
Goldstein, "Two-dimensional photonic crystal coupleddefect laser diode," Appl. Phys. Lett. 82, 4-6 (2003).

49. A. A. Savchenkov, V. S. Ilchenko, T. Handley, and L. Maleki, "Second-order filter response with series-coupled silica microresonators," IEEE Photonics Technol. Lett. 15, 543-544 (2003).

50. D. G. Rabus, M. Hamacher, U. Troppenz, and H. Heidrich, "High- $Q$ channel-dropping filters using ring resonators with integrated SOAs," IEEE Photonics Technol. Lett. 14, 1442-1444 (2002).

51. S. T. Chu, W. Pan, S. Sato, T. Kaneko, B. E. Little, and Y. Kokubun, "Wavelength trimming of a microring resonator filter by means of a UV sensitive polymer overlay," IEEE
Photonics Technol. Lett. 11, 688-690 (1999).

52. H. Haciwa, T. Naganawa, and Y. Kokubun, "Wide range center wavelength trimming of vertically coupled microring resonator filter by direct UV irradiation to SiN ring core," IEEE Photonics Technol. Lett. 16, 135-137 (2004).

53. S. T. Chu, B. E. Little, V. Van, J. V. Hryniewicz, P. P. Absil, F. G. Johnson, D. Gill, O. King, F. Seiferth, M. Trakalo, and J. Shanton, "Compact full $C$-band tunable filters for $50 \mathrm{GHz}$ channel spacing based on high order micro-ring resonators," in Optical Fiber Communication Conference (OFC), Vol. 95 of OSA Trends in Optics and Photonics Series (Optical Society of America, Washington, D.C., 2004), paper PDP9. 